\title{
Rheuma, Nierensteine, Darmbeschwerden - wie qualifizierter Ultraschall Erkrankungen sichtbar macht
}

\section{Bericht zur DEGUM-Pressekonferenz am 20. April in Berlin}

Welche modernen Möglichkeiten bietet Ultraschall, um Magen-Darm-Erkrankungen erkennbar zu machen? Und welche wichtigen Hinweise liefert das bildgebende Verfahren bei rheumatischen Erkrankungen? Warum sollte die Sonografie bei der Diagnose von Nierensteinen das Mittel der ersten Wahl sein? Über diese und weitere Fragen diskutierten Experten im April 2017 auf einer Pressekonferenz der Deutschen Gesellschaft für Ultraschall in der Medizin e. V. (DEGUM) in Berlin.

Am Anfang der Konferenz berichtete Professor Dr. med. Johannes Strunk, Chefarzt der Klinik für Rheumatologie im Krankenhaus Porz am Rhein in Köln und DEGUMKursleiter Stufe II, darüber, wie Rheuma per Ultraschall sichtbar gemacht werden kann. Etwa 1,5 Millionen Erwachsene in Deutschland leiden an der chronischen Krankheit. Sie kann zwar nicht geheilt werden, ein Leben ohne Einschränkungen ist aber möglich, wenn eine frühe Diagnose erfolgt. „Der Ultraschall ist dabei ein wichtiges Instrument“, sagte Strunk. „Er bietet gute Möglichkeiten die Krankheit rechtzeitig zu erkennen. So kann der Entwicklungsprozess bei vielen Patienten eingedämmt werden."

\section{Möglichkeiten des}

\section{Ultraschalls bei \\ Blindarmentzündung und Morbus Crohn}

Anschließend referierte Dr. med. Hans Worlicek, der Leiter der Kommission für Ultraschall in der Praxis der DEGUM ist und an der Gastroenterologischen Gemein- schaftspraxis am Facharztzentrum Regensburg tätig war, über den Einsatz des Ultraschalls bei Erkrankungen des Magen-DarmTrakts. „Eine krankhafte Wandverdickung des befallenen Darmabschnitts kann bei chronisch-entzündlichen Darmerkrankungen per Sonografie sehr genau dargestellt werden. Der Ultraschall liefert also wertvolle Hinweise, welche Krankheit vorliegt“, erläuterte Worlicek. „Die Klärung der Diagnose ist oft eine große Erleichterung, da der Leidensdruck vieler Betroffener hoch ist." Auch bei Blinddarm- und Divertikelentzündungen sei die Sonografie ein sehr wertvolles Instrument. So können die Erkrankungen auf diese Weise mittlerweile mit einer Genauigkeit von 95 Prozent erkannt werden.

\section{Nierensteine - warum Ultraschall das Instrument der ersten Wahl sein sollte}

Ein weiteres zentrales Thema auf der Pressekonferenz war die Sonografie bei Nierensteinen. Nach Ansicht von Professor Dr. med. Thomas Enzmann, stellvertretender Leiter der DEGUM Sektion Urologie und Chefarzt der Klinik für Urologie und Kinderurologie am Städtischen Klinikum Brandenburg, kommt diese in der Praxis zu selten zum Einsatz. „Rettungsstellen und Krankenhäuser, die keine Urologen oder keinen Arzt mit einer ausreichenden Ultraschallausbildung zur Verfügung haben, „schieben“ den Patienten zunächst durch einen Computertomografen“, so Enzmann. „Dadurch werden Betroffene jedoch mit einer Strahlenbelastung konfrontiert. Der Ultraschall kann bei der Diagnose von Nierensteinen sehr genaue Erkenntnisse lie- fern." Zudem sei er schnell durchzuführen und ist überall verfügbar.

\section{Sonografie der Bauchorgane}

- höhere finanzielle

Unterstützung durch Krankenkassen gewünscht

Auch ein gesundheitspolitisches Thema und zwar die finanzielle Unterstützung der Ultraschallmethode bei Erkrankungen des Bauchraumes - stand auf der Agenda. „89 Prozent der sonografisch tätigen Gastroenterologen schätzen die Finanzierung der Sonografie in der gesetzlichen Krankenversicherung als defizitär ein“, beklagte Worlicek. „Eine angemessene Finanzierung bleibt der hochqualifizierten, spezialisierten Bauchsonografie seit drei Jahrzehnten verwehrt. Und das trotz der unbestrittenen Effizienz." So sei es mittlerweile ein etabliertes Verfahren, die Kontrastmittel-Sonografie (CEUS) zur Tumordifferenzierung und Metastasensuche in der Leber sowie beispielsweise in der Diagnostik von Pankreas, Milz, Darm einzusetzen. Es handele sich hierbei um ein etabliertes Verfahren, dessen Einsatz in den deutschen S3-Leitlinien und den europäischen Ultraschall-Leitlinien gefordert würde. „Diese Methode wird jedoch nicht vergütet, obwohl zur Durchführung eine sehr teure technische Ausstattung notwendig ist und ausreichend qualifiziertes Personal vorhanden sein muss. Hier wünschen wir uns dringend eine Unterstützung von der gesetzlichen Krankenversicherung“, sagte der DEGUMExperte abschließend. 